\title{
Multum non multa: airway distensibility by forced oscillations
}

\author{
Charalampos Mermigkis1,2, Sophia E. Schiza², Panagiotis Panagou ${ }^{1}$ \\ 1 Pulmonary Department, 401 General Army Hospital, Athens \\ 2 Department of Thoracic Medicine, University General Hospital, Medical School of the University of Crete, \\ Greece
}

\begin{abstract}
Airway distensibility although appears to be unaffected by airway smooth muscle tone probably related to airway remodelling, after bronchodilator treatment is significantly increased in subjects with asthma. We assessed airway distensibity and its first moment derivative in two patients with mild intermittent asthma and normal spirometry. The increase in airway distensibility after bronchodilation measured at the tidal volume range during quiet breathing by forced oscillations was not accompanied by a change in its first moment, while the latter showed a significant increase in a second patient after anti-inflammatory treatment. It appears that airway distensibility is sensitive to reduction of bronchial smooth muscle tone after bronchodilation, but in addition its first moment might provide information on a change of both bronchial smooth muscle tone and small airways inflammation.
\end{abstract}

\section{Introduction}

Forced oscillometry is probably a technique that could be included in routine clinical practice in order to provide useful data related to the underestimated severity of small-airway obstruction in bronchial asthma and bronchial hyper-responsiveness (BHR) .

The derivative, or first moment of airway distensibility (DD), assessed as the linear regression slope of conductance versus lung flow, is not currently measured in routine clinical practice, even though it could provide crucial clinical information concerning the different effects of bronchial smooth muscle tone and mucosal inflammation on airway caliber.

Corresponding author: Charalampos Mermigkis, Pulmonary Department, 401 General Army Hospital, Athens, Greece. E-mail: mermigh@gmail.com

Key words: Forced oscillations; impedance; airway distensibility; small airways; admittance.

Conflict of interests: the authors have no conflicts of interest.

Received for publication: 17 February 2014

Accepted for publication: 5 May 2014

(C) Copyright C. Mermigkis et al., 2015

Tipografia PI-ME Editrice, Italy

Monaldi Archives for Chest Disease Pulmonary Series 2015; $81: 746$

doi: 10.4081/monaldi.2015.746

This article is distributed under the terms of the Creative Commons Attribution Noncommercial License (by-nc 4.0) which permits any noncommercial use, distribution, and reproduction in any medium, provided the original author(s) and source are credited.
Our hypothesis was that in the former case first moment DD would not change significantly, whereas it would show an analogous change in the latter case. We present a clinical example of two patients treated in our department who reflected these different conditions.

As research in forced oscillation physiology is an ongoing hot topic, studies incorporating and assessing the value of new indices in BHR and small-airway physiology in general might prove clinically useful.

\section{Case report}

Performing studies with provocation tests with small airways measurements, e.g. impulse oscillometry, and small-particle stimuli will give better insights into BHR of the small airways, but little is known about the severity of small airways obstruction and the severity of BHR [1]. Oscillometry provides analysis of intrabreath mechanics and recently airway distensibility has been measured (as the relationship of the conductance-lung volume relationship) and found to be more sensitive in assessing bronchodilator responsiveness in relation to reactance $v s$ lung volume [2]. However because of the known effect of flow on resistance [3], the first moment of airway distensibility, assessed as the conductance-lung flow relationship and its change after bronchodilation or anti-inflammatory treatment has not been assessed to date, although it might provide information regarding the behavior of small airways to change.

\section{Case 1}

Asymptomatic patient with chronic intermittent asthma. Normal spirometry (flow-volume curve) after maximum effort: $\mathrm{FEV} 1=80,7 \%$, FEV1/FVC $=81 \%$, FEF25-75=72,6 (pred). Forced oscillations of the same patient (before and after inhalation of $400 \mu \mathrm{gr}$ salbutamol), were performed (coherence function $>0.90$ ), IOS System Jaeger (Figure 1 and Table 1): An increase of Resistance R(rs5), Reactance at $5 \mathrm{~Hz}$ (X(rs5), of resonant frequency (RF) and a negative frequency dependence is found. After bronchodilation: reduction of Reactance area (a sensitive index of small airways, $\mathrm{AX}$ ): $25 \%$. Changes in conductance (reciprocal of resistance) at $5 \mathrm{~Hz}$ (G (rs5)) were related to changes in lung volume, linear regression slope of $\Delta \mathrm{G}(\mathrm{rs} 5) / \Delta \mathrm{V}(\mathrm{L})$, an index of airway distensibility and showed relatively greater change (increase) of airways distensibility, (cm H20-1 * $\mathrm{sec}^{-1}$ ) of $34 \%$, which is explained by a reduction of bronchial smooth muscle tone because of a beta agonist effect and not to anti-inflammatory action (fall of R(rs5), X(rs5), AX, RF, but persistence of negative frequency dependence) [4]. However the derivative of distensibility (first moment), linear regression slope of $\Delta \mathrm{G}(\mathrm{rs} 5) / \Delta$ flow, $\mathrm{cmH}_{2} \mathrm{O}$ $1^{*}\left(\sec ^{-1}\right)[2]$, showed an insignificant change of $2.8 \%$.

\section{Case 2}

A patient with mild asthma exacerbation, after using for 12 weeks inhaled steroid therapy, showed similar changes as above (difference 


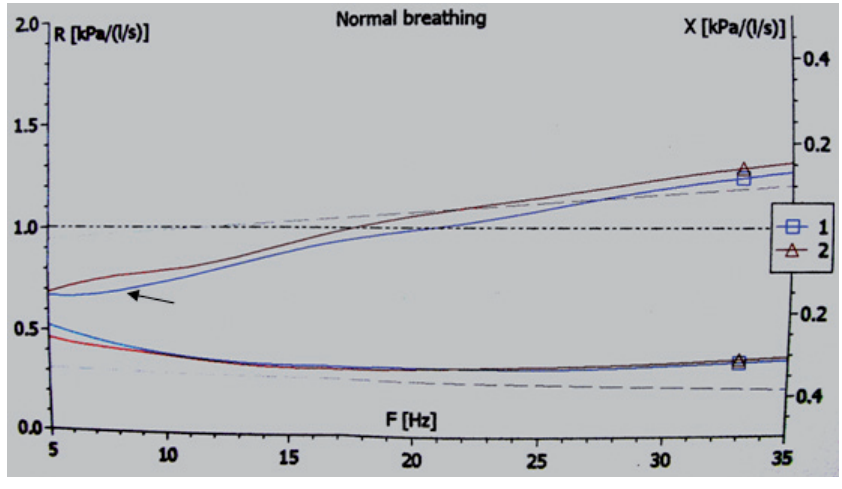

Figure 1. Forced oscillations graph (descriptions in text).

Table 1. Oscillometric data before and after bronchodilation.

\begin{tabular}{lccc} 
Pre B/d & $\%$ pred. & Post B/d & $\%$ Difference \\
Zrs 5Hz: 0,55 [kPa/(L/s)] & 175,5 & 0,49 & $-11 \%$ \\
\hline Rrs 5Hz: 0,52 [kPa/(L/s)] & 167,9 & 0,46 & $-11,6 \%$ \\
\hline Xrs 5Hz: -0,17 [kPa/(L/s)] & 587,0 & $-0,16$ & $-4,6 \%$ \\
\hline AX: $1,40[\mathrm{kPa} / \mathrm{L}]$ & & 1,06 & $-24,8 \%$ \\
\hline Resonant Frequency: 20,83 [1/s] & & 17,69 & $-15,1 \%$ \\
\hline
\end{tabular}

from baseline), but also the negative frequency dependence was reduced (graph not shown). Airways distensibility and its first moment showed much greater and quasi- similar changes, probably reflecting a reduction of both bronchial airway smooth muscle tone and inflammation ( $97 \%$ and $46 \%$ respectively).

\section{Discussions and conclusions}

Forced oscillation technique (FOT) offers a simple and detailed approach to investigate the mechanical properties of the respiratory system. The method is simple, requires no patient cooperation and no forced expiratory maneuvers. The information provided by FOT is considered complimentary to spirometry [5]. In this study, for the first time, airway distensibility and its first moment were assessed in patients with mild intermittent asthma having normal spirometry. We found that airway distensibility was more sensitive to bronchodilation in relation to other oscillometric indices. When airway inflammation is present, then airway distensibility along with its first moment probably might better reflect the change of flow resistive properties of small airways.
Brown et al. found that airway distensibility is unchanged after bronchodilation and probably reflects airway remodeling in asthma [6]. However their methodology included measurements at the TLC or near the TLC range and it is known that elevated airway tone causes airway constriction which may be overcome by a deep breath and to the progressive insensitivity of airway distensibility to airway smooth muscle tone at higher lung volumes (curvilinear relationship).

In this study we assessed the effect of elevated airway smooth muscle tone around FRC (linear portion of the conductance-volume diagram), which causes reduction of airway compliance and stiffer lungs in asthma. Reduction of airway tone by bronchodilation alters airway distensibility by FOT by decreasing lung elastance, so, in real life situations as in the present study, alterations in airway distensibility reflect bronchial tone and peripheral mechanical tissue properties $[6,7]$.

The findings of our study in mild intermittent asthma patients are in agreement to the data of Kelly et al. concerning changes of airway distensibility and respiratory system reactance [2] (there is practical no difference between $5-8 \mathrm{~Hz}$ oscillations protocol). The validity and usefulness of the first moment of airway distensibility as a more sensitive index of a significant change in small airway pathology should be corroborated in future larger prospective studies.

\section{References}

1. Telenga ED, den Berge M, Hacken NHT, et al. Small airways in asthma: their independent contribution to the severity of hyperresponsiveness. Eur Respir J 2013;41:752-4.

2. Kelly VJ, Brown NJ, Sands SA, et al. Effect of airway smooth muscle tone on airway distensibility measured by forced oscillation technique in adults with asthma. J Appl Physiol 2012;112: 1494-503.

3. Marchal F, Loos N, Monin P, Peslin R. Methacholine-induced volume dependence of respiratory resistance in preschool children. Eur Respir J 1999;14:1167-74.

4. Smith HJ, Reinhold P, Goldman MD. Forced oscillation technique and impulse oscillometry. Eur Respir Mon 2005;31:72-105.

5. Oostven E, Mac Leod D, Lorino H, et al. The forced oscillation technique in clinical practice: methodology, recommendations and future developments. Eur Respir J 2003;22:1026-41.

6. Brown NJ, Salome Cm, Berend N, et al. Airway distensibility in adults with asthma and healthy subjects measured by forced oscillation technique. Am J Respir Crit Care Med 2007;176:129-37.

7. Kaczka DW, Ingenito EP, Israel E, Lutchen KR. Airway and lung tissue mechanics in asthma. Effect of albuterol. Am J Repir Crit Care Med 1999;159:169-75. 